\title{
LEISHMANIOSE CUTÂNEA ACIDENTAL
}

\author{
Neuza Lima Dillon (1), Hamilton OMETTO STOLF (2), Elizaide L. ALVARENGA YOSHIDA (3) \& \\ Mariangela E. ALENCAR MARQUES (4)
}

\begin{abstract}
RESUMO
Os autores relatam o $3^{\circ}$. caso de Leishmaniose acidental em laboratório, ocorrido em aluna do curso de Biologia, que contaminou-se por ocasião da passagem de formas amastigotas de Leishmania (Viannia) braziliensis de hamster infectado para hamster são.

Chamam atenção de que, apesar de usar toda proteção exigida, a aluna foi mordida pelo hamster são, havendo como conseqüência, ruptura da luva e contágio através do inóculo.
\end{abstract}

UNITERMOS: Leishmaniose cutânea; Leishmania (Viannia) braziliensis infecção acidental.

\section{INTRODUÇÃO}

Leishmaniose acidental por infecção em laboratório com Leishmania donovani foi relatada por TERRY et al. em $1950^{2}$. SAMPAIO et al. em $1983^{1}$ relataram fato semelhante ocorrendo em estudante do curso de Biologia, que infectou-se com material de cultura de Leishmania braziliensis braziliensis.

\section{RELATO DO CASO}

Recentemente compareceu ao serviço de Dermatologia da Faculdade de Medicina de Botucatu - UNESP, aluna do curso de Biologia, referindo que durante o procedimento de passagem de formas amastigotas de hamster infectado com Leishmania (Viannia) braziliensis para hamster normal (repique), foi mordida pelo hamster normal, rompendo-se a luva e contaminando-se com o inóculo, apesar de estar adequadamente paramentada.

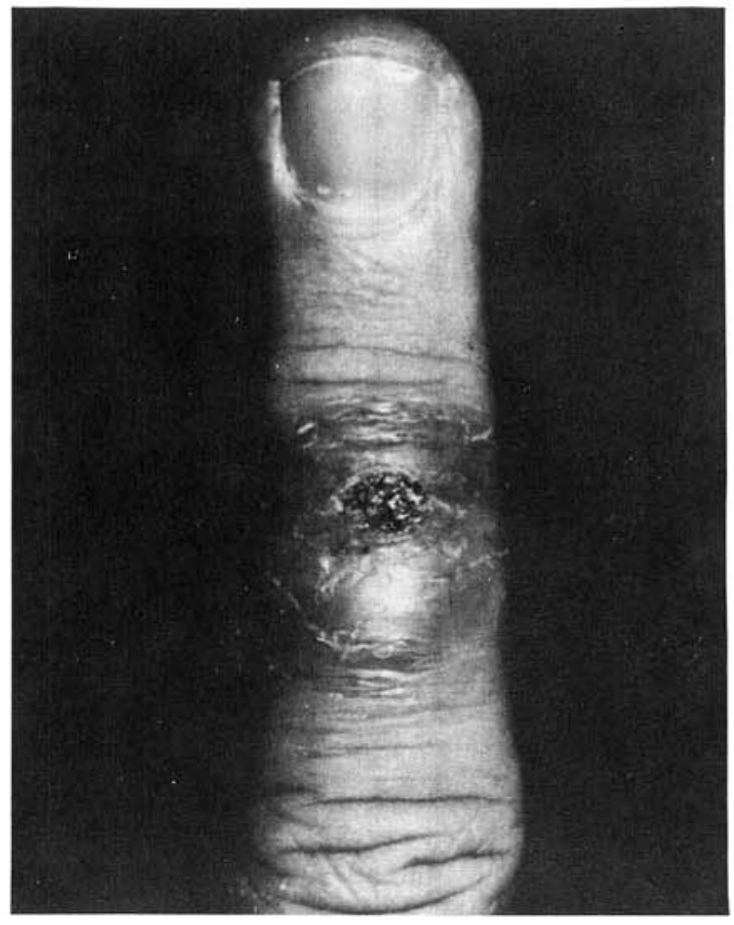

Figura 1 - Lesão nódulo-ulcerada. Dois meses após o acidente.

(1) Profa. Titular do Departamento de Moléstias Infecciosas e Parasitárias, Dermatologia e Radiologia da Faculdade de Medicina de Botucatu - UNESP.

(2) Prof. Assistente do Departamento de Moléstias Infecciosas e Parasitárias, Dermatologia e Radiologia da Faculdade de Medicina de Botucatu - UNESP.

(3) Profa. Assistente Doutora do Departamento de Parasitologia do Instituto de Biociências de Botucatu - UNESP.

(4) Profa. Assistente do Departamento de Anatomia Patológica da Faculdade de Medicina de Botucatu - UNESP.

ENDEREÇO PARA CORRESPONDÊNCIA: Neuza Lima Dillon, Disciplina de Dermatologia, Faculdade de Medicina de Botucatu, UNESP. CEP: 18.618-000, Botucatu, São Paulo, Brasil. 
Referia que no local da mordida, dedo médio da mão esquerda, houve cicatrização parcial da lesão com persistência de pequena pápula. Posteriormente, piora e, após dois meses, apresentava lesão nódulo-úlcerada com linfangite ascendente, superfície eritematosa viva com aumento de temperatura, dor espontânea e à palpação (figura 1). O diagnóstico proposto foi o de erisipela, sendo medicada com antibiótico sistêmico.

Três meses após, sem melhora, foi feito diagnóstico de esporotricose, passando a receber $6,0 \mathrm{~g}$ de iodeto de potássio ao dia, durante 5 meses.

Aos 10 meses, após tratamento infrutífero com iodeto de potássio, compareceu a este serviço apresentando na face dorsal do dedo médio e mão esquerda, numerosas pápulas de superfície eritematosa, infiltradas, isoladas ou confluentes, medindo de 1 a $8 \mathrm{~mm}$ de diâmetro, dispostas ou não sobre área de pele eritemato-infiltrativa, com aumento de volume do dedo (figura 2).

Foi sugerido o diagnóstico clínico de leishmaniose e confirmado através de exame histopatológico (figura 3)

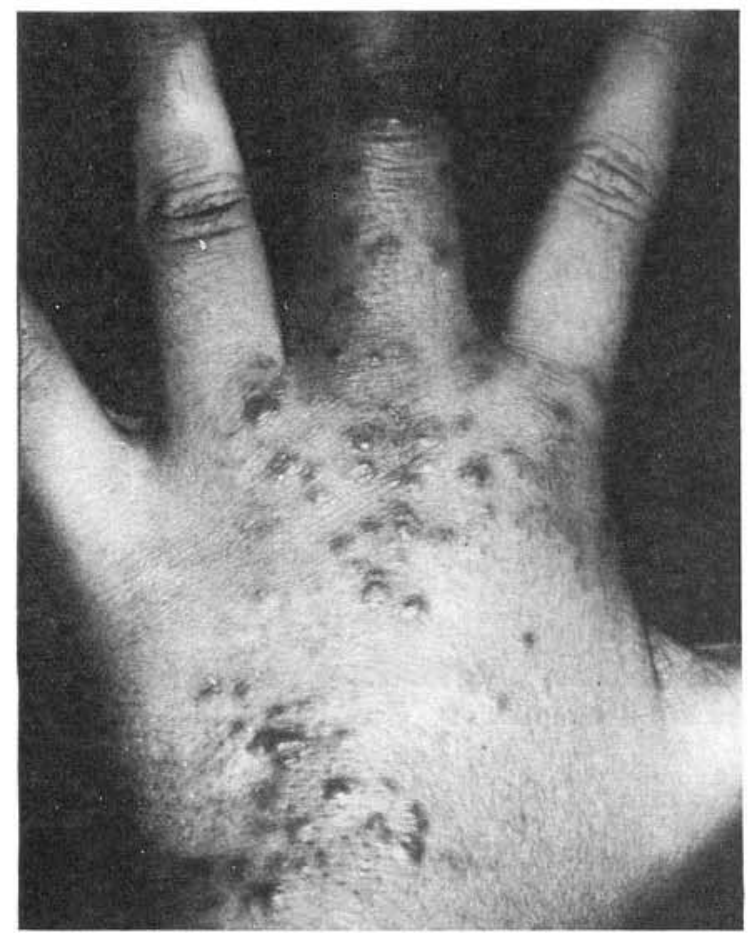

Figura 2 - Múltiplas lesões papulosas. Dez meses após o acidente.

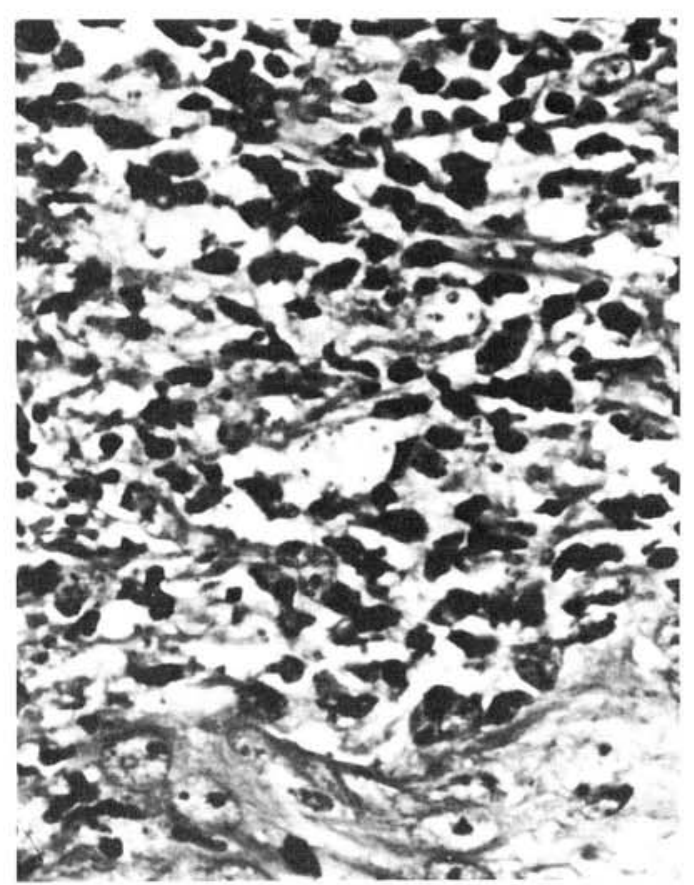

Figura 3 - Histopatologia evidenciando ninhos de Leishmania. H. E. $400 x$.

Medicada com antimoniato de N-metilglucamina, 2 séries de 20 ampolas intervaladas de 10 dias e associado prednisona $20 \mathrm{mg}$ ao dia quando da segunda série do medicamento. Com esse tratamento houve regressão total das lesões (figura 4).

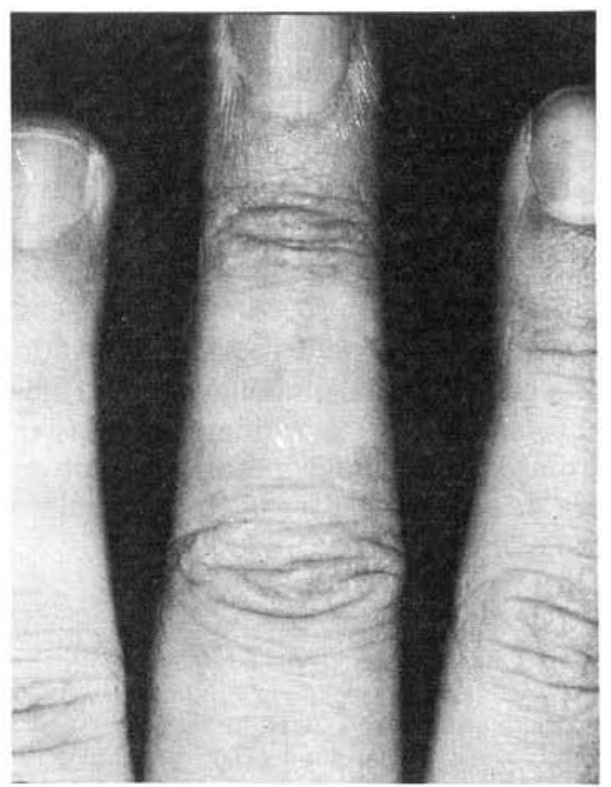

Figura 4 - Regressão das lesões, dois meses após tratamento com o antimoniato de N-metil-glucamina. Curada. 


\section{COMENTÁRIOS}

Infecções acidentais com leishmania foram primeiramente referidas por TERRY et al. em $1950^{2}$ por Leishmania donovani e SAMPAIO et al. ${ }^{1}$ por Leishmania braziliensis.

Os autores consideram de importância o relato deste caso devido ao pequeno número de casos na literatura e pela importância do relato de contaminação em laboratório, pois normas rígidas de segurança e de treinamento prévio e intensivo podem ser rompidas e o acidente ocorrer. No laboratório de Parasitologia do Instituto de Biologia da UNESP é rotina usar avental cirúrgico, comprido, grossas luvas e capacete para a proteção facial, pois em trabalhos com Leishmania, Trypanosoma, Toxoplasma e outros, existe o risco potencial de infecções acidentais.

\section{SUMMARY \\ ACCIDENTAL CUTANEOUS LEISHMANIASIS}

The authors report the $3^{\text {rd }}$ case of cutaneous leishmaniasis due to accidental contamination of a student of biological sciences during laboratory research.

The inoculation ocurred when amastigotes of Leishmania (Viannia) braziliensis from infected hamsters were injected in healthy animals. At this stage, the student had her glove bitten by a healthy hamster and the infection was probably conveyed by the direct contact of the inoculum with the skin.

\section{REFERÊNCIAS BIBLIOGRÁFICAS}

1. SAMPAIO, R. N.; DE LIMA, L. M. P.; VEXENAT, A.; CUBA, C. C.; BARRETO, A. C. \& MARDEN, P. D. - A laboratory infection with Leishmania braziliensis braziliensis. Trans. roy. Soc. trop. Med. Hyg., 77: 274, 1983.

2. TERRY, L. L.; LEWIS, J. L. \& SESSONS, S. M. - Laboratory infection with Leishmania donovani: a case report. Amer. J. trop. Med., 30: 643-649, 1950.

Recebido para publicação em 04/12/1992 Aceito para publicação em 27/04/1993 